\title{
161 AN EXTENDED CLIPPERS PHENOTYPE
}

Christopher Lane, ${ }^{1,2}$ Robin Howard, ${ }^{1,2}$ Rahul Phadke $2 .{ }^{1}$ Guy's and St. Thomas' NHS Trust, ${ }^{2}$ National Hospital, Queen Square

10.1136/jnnp-2014-309236.161

CLIPPERS (chronic lymphocytic inflammation with pontine perivascular enhancement responsive to steroids) is a recently described relapsing-remitting CNS inflammatory condition which predominantly affects the brainstem. We present a patient with features consistent with the syndrome who re-presented with seizures (not previously reported) and a space occupying lesion on imaging. Initially a diagnosis of metastatic carcinoma was made in the referring hospital. Due to diagnostic uncertainty cerebral biopsy was performed showing a densely packed lymphohistiocytic infiltrate consistent with CLIPPERS. Following a further brainstem relapse the patient was treated with high-dose steroids, subsequently switched to a tapering regime. She did not tolerate a second line immunosuppressant but remains well on steroids.

This case demonstrates an extended CLIPPERS imaging and clinical phenotype with prominent cortical involvement 
resulting in seizures. The diagnosis is one of exclusion and the case serves to emphasise that cerebral biopsy, although invasive, is an important investigation in cases of diagnostic uncertainty allowing exclusion of other inflammatory, vasculitic or neoplastic conditions. 